EDITORIAL

Editor-in-Chief

\title{
REDCap: web-based software for all types of data storage and collection
}

\author{
L. A. Harvey ${ }^{1}$
}

( ) International Spinal Cord Society 2018

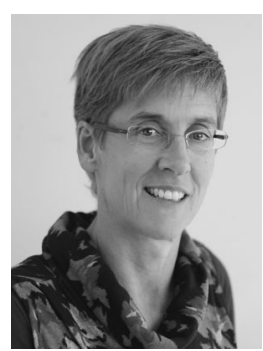

Gone are the days of entering research data into Excel spreadsheets or paying IT specialists to build databases for data registries or specific trials and projects. These systems of data management have been replaced by Research Electronic Data Capture (REDCap) Software [1, 2], as referred to in an interesting paper in this edition of Spinal Cord about data registries for canines [3].

The REDCap software was developed by a small group of academics who were committed to helping all researchers collect and store their data in a secure and fully transparent way. Importantly, this software is now used by a consortium of over 2,600 non-for-profit institutions. The consortium was launched in 2006. While the software is free, it is not open-source or freeware software. So an individual cannot download the software. Instead, users need to be part of an institution that joins the consortium. But there are no hidden costs. Any institution can join the consortium at no charge provided they attain an end-user license agreement. Institutions require some infrastructure to support the software (eg. PHP web server, MySQL database server) and need to provide some technical expertise for installing and maintaining the software. However, once up and going, an institution probably only needs one person to support hundreds of REDCap users.

REDCap has many, many features which should be of great interest to researchers, particularly those involved in collecting clinical data and developing patient registries [4]. It is primarily a customisable data collection tool with a userfriendly data entry interface. Users can be set up with different access privileges according to their roles in a study. It is especially useful for clinical trials because it can manage the randomisation procedures of participants in a concealed but fully traceable way. It also accommodates double-data entry; essential for minimising data entry errors. It has a formal data querying interface so that any data point can be

\footnotetext{
L. A. Harvey

spinalcord@iscos.org.uk

1 University of Sydney, Sydney, Australia
}

queried with a fully transparent record of decisions or changes in data. REDCap contains a sophisticated tracking system so that every users' actions are fully recorded. This creates a complete audit trail thereby preventing data from being accidentally or intentionally changed. Similarly, it prevents files with the latest version of data being mistakenly overwritten or files being corrupted. In addition, REDCap enables users to store scanned hard copies of Case Report Forms (used for data collection) on the database. REDCap also contains the capacity to collect data from participants through online surveys not dissimilar to the commonly used Survey Monkey software. A REDCAP database housed at one institution can be accessed by anyone worldwide provided the user is approved by a local administrator. Once a survey or study is complete, the database can be locked so that no inadvertent changes are made.

Statisticians have embraced REDCap because its data can be downloaded and directly used in most of the common statistical programs (eg. SAS, STATA and SPSS). In all, Spinal Cord encourages its researchers to consider REDCap for the management of data. It provides a step in the right direction to help ensure transparent data collection that is fully traceable. This is important for ensuring research integrity.

\section{References}

1. https://projectredcap.org/about/. Accessed on June 22, 2018.

2. Wright A. REDCap: A Tool for the Electronic Capture of Research Data. J Electron Resour Med Libr. 2016;13:197-201.

3. Moore SA, Zidan N, Spitzbarth I, Nout-Lomas YS, Granger N, da Costa RC, et al. Development of an International Canine Spinal Cord Injury (CSCI) observational registry: a collaborative datasharing network to optimize translational studies of SCI. Spinal Cord. 2018;56:656-665.

4. Pang X, Kozlowski N, Wu S, Jiang M, Huang Y, Mao P, et al. Construction and management of ARDS/sepsis registry with REDCap. J Thorac Dis. 2014;6:1293-99. 